\title{
Cardiovascular Manifestations of HIV Infection- A Review
}

\author{
Kshitiz Nath ${ }^{1}$, Lakhan Singh ${ }^{2}$ \\ ${ }^{1}$ Department of General Medicine, Maharani Laxmi Bai Medical College, Jhansi, Uttar Pradesh, India. \\ ${ }^{2}$ Department of General Medicine, Maharani Laxmi Bai Medical College, Jhansi, Uttar Pradesh, India.
}

\section{ABSTRACT}

\section{BACKGROUND}

HIV is a single-stranded RNA retrovirus from the Lentivirus family that invades cells containing specific membrane receptors and incorporates a DNA copy of itself into the host's genome. Immune deficiency is the result of virus and immune-mediated destruction of CD4 lymphocytes caused by continuous high-level HIV replication. AIDS is defined by the development of one or more specified opportunistic infections, tumours, and other conditions. These include oesophageal candidiasis, cytomegalovirus CMV retinitis, pulmonary or extrapulmonary tuberculosis, Kaposi sarcoma, and HIV/AIDS associated dementia. Most forms of HIV-related heart muscle disease and pericardial effusion occur at this stage. Acquired immunodeficiency syndrome (AIDS) was first recognized in 1981 and is caused by human immunodeficiency virus (HIV-1). HIV-2 causes a similar illness to HIV-1 but is less aggressive and has so far been restricted mainly to western Africa. HIV/AIDS is acquired through exposure to infected body fluid, particularly blood and semen; the most common modes of spread are sexual, parenteral (blood or blood product recipients, injection drug users, and occupational injury) and vertical (mother to fetus). HIV/AIDS is now the second leading cause of death in the world, with a global prevalence of $0.8 \%$.

Many cultural and social factors determine regional patterns of HIV/AIDS disease and associated infections. ${ }^{1}$ In the United States and northern Europe, the epidemic has predominantly been in men who have sex with men. In northeast India, the incidence has been greatest in injection drug users, but in much of Southeast Asia, the dominant routes of transmission have been heterosexual and from mother to child (vertical). With improving longevity, non-AIDS conditions are now accounting for the majority of deaths among individuals receiving ART, and CV disease has become an increasingly significant problem in the HIV population. Deaths due to $\mathrm{CV}$ disease among individuals living with HIV have ranged from $6.5 \%$ to $15 \%$ of total deaths. The mechanisms underlying CV disease in HIV patients are largely poorly understood but are known to be multifactorial. They include many traditional risk factors and also factors related to HIV, such as the side effects of antiretroviral medication. These effects are significant and include metabolic issues, immune activation, chronic inflammation, microbial translocation, and co-infection with other viral pathogens such as cytomegalovirus. Such mechanisms are ongoing even when HIV infection has been treated; the CD4 count and HIV viral load may be controlled, but the infection has not been cured. At the beginning of the epidemic, heart muscle disease (cardiomyopathy) was the dominant cardiac complication of HIV infection in the developed world. In contrast, tuberculous pericarditis and cardiomyopathy were and still remain important cardiac manifestations of the disease in Africa. ${ }^{2,3}$ Combined highly active antiretroviral therapy (HAART) (usually two nucleoside reverse transcriptase inhibitors [NRTIs] in combination with one or two protease inhibitors) has changed the pattern of disease in developed countries, where premature coronary artery disease (CAD) and other manifestations of atherosclerosis are emerging as the most common cardiovascular disorder.

\section{KEY WORDS}

HIV, Pulmonary Hypertension, Coronary Heart Disease, Pericardial Effusion, Myocarditis
Corresponding Author:

Kshitiz Nath,

Department of General Medicine

Maharani Laxmibai Medical College,

Jhansi, Uttar Pradesh, India.

E-mail:dr.kshitiznath@gmail.com

DOI: $10.14260 / j e m d s / 2020 / 262$

Financial or Other Competing Interests: None.

How to Cite This Article:

Nath K, Singh L. Cardiovascular manifestations of HIV infection- a review. J. Evolution Med. Dent. Sci. 2020;9(14): 1208-1214, DOI

10.14260/jemds/2020/262

Submission 30-01-2020,

Peer Review 13-03-2020,

Acceptance 21-03-2020,

Published 06-04-2020.

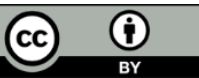




\section{BACKGROUND}

This is partly attributable to HAART-induced metabolic problems, particularly insulin resistance and hyperlipidaemia, but also reflects a high prevalence of conventional cardiovascular risk factors such as smoking. Cardiovascular problems associated with advanced immunodeficiency, such as heart muscle disease, pericardial effusion, and pulmonary hypertension, continue to predominate in resource-poor countries, where fewer than $5 \%$ of patients have access to antiretroviral drugs. ${ }^{2,3}$

\section{Coronary Heart Disease}

Although HAART clearly has beneficial effects on both mortality and morbidity in HIV/AIDS, concern remains over the potential metabolic side effects and the possibility of an epidemic of iatrogenic premature atherosclerotic disease in these patients. The first reports of acute MI in treated HIV/AIDS patients emerged in the late 1990s. It is now recognized that HIV/AIDS patients are at significantly greater risk of MI and coronary heart disease (CHD) than uninfected people of a similar age. Compared with national registries of non-HIV/AIDS patients, CHD in HIV/AIDS patients occurs mostly in men and at a younger age, suggesting that the ageadjusted incidence may be significantly higher. ${ }^{4}$ The incidence of CHD appears to be up to three times the incidence of a general male population and results in higher rates of acute MI and hospitalization for ACS compared with non-HIV/AIDS patients. There is a intriguing possibility that opportunistic infections such as CMV or Chlamydiae pneumoniae may play a role in the development of accelerated atherosclerosis. ${ }^{5}$ However, ACS are not clearly related to HIV replication because HIV RNA may be undetectable in up to one-third of patients at the time of presentation. Traditional risk factors such as cigarette smoking and diabetes mellitus etc. in this patient group should not be overlooked and HIV/AIDS patients may be at higher risk of developing hypertension at a younger age than the general population. However, accelerated atherosclerosis has also been observed in HIV/AIDS patients without traditional risk factors for CHD. Acute MI appears to be the most common presentation of CHD, and a high ratio of acute MI to stable angina exists in HIV/AIDS populations. ${ }^{4}$ Because these ACS involve lipid-rich plaques, the metabolic syndrome of HIV/AIDS lipodystrophy may promote the development of vulnerable lesions or influence important plaque rupture. However, it remains difficult to establish an absolute link between protease inhibitor exposure and CHD, and further long-term studies are required. Coronary angiography may be carried out in HIV/AIDS patients safely and frequently reveals patterns of coronary disease similar to young nonHIV/AIDS patients, specifically proximal vessel involvement and single-vessel disease. ${ }^{4}$ Treatment with percutaneous coronary intervention (PCI), fibrinolysis, and coronary artery bypass are all described with reasonable survival rates, although restenosis after

PCI is unexpectedly high and it has been associated with higher C-reactive protein levels and higher levels of CD8+ T cells. It therefore seems reasonable that the clinical situation should determine the use of invasive and non-invasive coronary investigations as for the non-HIV/AIDS population.

\section{Cardiomyopathy and Left Ventricular Abnormalities}

Dilated cardiomyopathy as a complication of HIV infection was first described in 1986. Isolated LV dysfunction in HIV/AIDS patients may resolve spontaneously, suggesting a self-limiting myocarditis. The differential diagnosis of cardiomyopathy in HIV/AIDS includes LV dysfunction secondary to ischemic heart disease, diabetes or hypertension, hypersensitivity reactions to drugs or foreign injected material, and coronary spasm secondary to cocaine use. The prevalence of heart muscle disease appears to be around $4.4 \%$ for cardiomyopathy and $6.4 \%$ for isolated $\mathrm{LV}$ dysfunction, and these conditions may cause symptoms in up to $5.5 \%$ of HIV/AIDS patients. ${ }^{6}$ Cardiomyopathy is associated with poor survival in HIV/AIDS patients.

\section{Mechanisms of Cardiomyopathy in HIV/AIDS}

The mechanisms for the development of LV dysfunction or cardiomyopathy and myocarditis in AIDS remain unclear. In addition to the role of HIV and its proteins, lymphocytic myocarditis and cytokines, the contributions of autoimmune responses, illicit and prescribed medications, nutritional deficiencies, and other factors also appear to be pathogenetically or pathophysiologically important.

Autoimmunity and HIV/AIDS-Related Heart Muscle Disease - Many autoimmune processes have been described in association with HIV/AIDS. Although some of these may be the result of opportunistic infection, HIV infection may trigger autoimmune phenomena in susceptible patients. The significance of some autoantibodies such as antineutrophil cytoplasmic autoantibody or antiphospholipid antibodies in HIV/AIDS remains unclear. However, the presence of such antibodies with hypergammaglobulinemia and elevated circulating immune complexes suggests that an as yet undefined autoimmune process may take place in HIVpositive patients. ${ }^{7}$ Autoantibodies against ?eta-myosin have been identified in HIV positive patients with cardiomyopathy and histologically proven active myocarditis. These findings support a possible autoimmune process in the pathogenesis of HIV/AIDS heart muscle disease. Common, cardiotropic viruses could facilitate development of cardiac autoimmunity in HIV-positive patients by modifying myocyte surface antigens. CMV infection is a common opportunistic infection in AIDS patients, but it has been described only infrequently as a cause of myocarditis in HIV/AIDS. Although this might suggest that CMV is not strongly implicated, in situ hybridization studies have identified transcripts of CMVspecific DNA within the myocytes of HIV-positive patients with myocarditis and cardiomyopathy in the absence of typical histologic features such as inclusion bodies. ${ }^{8}$ In this way, CMV or some other factors such as circulating HIV viral proteins could be responsible for the ongoing inflammation and cardiac injury seen in many cases of HIV/AIDS myocarditis. Cardiac dysfunction and heart muscle disease have been associated with the expression of HIV proteins Tat and Vpr in transgenic mice.

Drug-Induced Heart Muscle Disease- Both HIV-1 infection per se and antiretroviral therapy, particularly NRTIs, may have a negative impact on myocardial function. NRTIs are key elements of HAART, and zidovudine (azidothymidine) in particular has been implicated in the development of some cases of HIV-related heart muscle disease. In addition to 
inhibiting HIV reverse transcriptase, the drug causes a dosedependent reversible skeletal myopathy by altering mitochondrial DNA replication. Recent reports suggest that mitochondrial toxicity can also be caused by nucleoside analog therapy for hepatitis $\mathrm{B}$, a common coinfection with HIV-1, and makes treatment of both infections potentially more complex. The exact role of zidovudine in the pathogenesis of HIV-related heart muscle disease in man remains unclear, and evidence for its existence is limited; however, small numbers of HIV-positive patients who have developed cardiac dysfunction while taking the drug were seen to improve after its discontinuation. Other drugs used in the treatment of HIV/AIDS patients may also result in cardiac dysfunction. Foscarnet, indicated for CMV infection in adult AIDS patients, has been associated with episodes of reversible congestive cardiac failure as have doxorubicin and interferon-? ?therapy for Kaposi sarcoma. Cocaine use has been associated with myocarditis and a possibly reversible cardiomyopathy in non-AIDS patients and thus should be considered in HIV/AIDS patients.

Nutritional Deficiencies and Cardiac Dysfunction in HIV/AIDS- HIV/AIDS patients with evidence of LV systolic dysfunction should be assessed for micronutrient deficiency. Abnormally low levels of selenium and antioxidant vitamins in particular have been demonstrated, and "oxidative stress" may be an important mechanism for cellular damage in AIDS. Selenium deficiency is implicated in the pathogenesis of Keshan disease, a specific form of dilated cardiomyopathy. In the same way, decreased selenium content has been demonstration in the hearts of individuals with AIDS, and selenium replacement was associated with an improvement in LV dysfunction in a small group of HIV-positive patients. LCarnitine deficiency has also been described in HIV/AIDS patients, possibly in association with cardiac symptoms and in whom again supplementation may be advantageous.

\section{Echocardiographic Features of HIV/AIDS-Related Heart Muscle Disease}

The hallmark is global LV systolic dysfunction with the consistent feature of reduced ejection fraction. Examination of adults most frequently reveals a reduced or normal ventricular wall thickness in association with systolic dysfunction; in children, LV hypertrophy with cavity dilatation is well recognized. ${ }^{9}$ LV dilatation can result in mitral valve distortion and lead to regurgitation. This may contribute to secondary pulmonary hypertension and RV dilatation, although this more frequently occurs as part of the cardiomyopathic process, in isolation, or in association with pulmonary hypertension. Abnormalities of mitral flow, specifically reduced early mitral peak velocity and other indices of diastolic dysfunction, have been noted early in the course of HIV/AIDS with normal ejection fraction and in association with LV systolic dysfunction.

\section{Treatment of Left Ventricular Dysfunction in HIV/AIDS}

Evidence-based treatment may prove applicable to HIV/AIDS patients with respect to morbidity and mortality improvements. Therefore, despite a seemingly poor prognosis, HIV/AIDS patients with LV dysfunction should be offered appropriate treatment. If LV dilatation and hypokinesis are found with or without clinical evidence of heart failure, consideration should be given to stopping all drugs that are not considered absolutely essential. If a 2-week follow-up echocardiogram reveals improvement, any suspected causative drug should be eliminated if at all possible. Essentially the same armamentarium of heart failure drugs is available for use in HIV/AIDS patients with LV dysfunction as for the non-AIDS population. Common agents such as diuretics, aldosterone antagonists, and digoxin may bring about symptomatic improvement. Angiotensinconverting enzyme inhibitors however, may be poorly tolerated, possibly because many patients may already have a low systemic vascular resistance. Etanercept, a TNF antagonist, and pentoxifylline, a TNF-? inhibitor, have been used in non-HIV patients with severe heart failure with some success. Intravenous immunoglobulin therapy has been used successfully in children with symptomatic HIV/AIDS heart muscle disease. Case reports document successful use of LV assist devices and orthotopic heart transplant exist, although these remain uncommon treatments in this population.

\section{Arrhythmias and Sudden Cardiac Death}

Sudden death and rhythm abnormalities are common in HIV infection and account for up to $20 \%$ of cardiac related deaths in this group of patients. These may be secondary to other cardiac pathology or be a consequence of some forms of treatment. For example, pentamidine, which is used in the treatment and prophylaxis of $P$. carinii infection, is structurally similar to procainamide and may cause torsade de pointes ventricular tachycardia when used intravenously or intramuscularly. Ganciclovir, an acyclic nucleoside used in the treatment of severe CMV infection, may also be arrhythmogenic. Concomitant electrolyte disturbance may be important in the development of cardiac arrhythmia, and careful evaluation of the QT interval and magnesium concentration should be used as a guide to cardiac toxicity. ECG abnormalities and rhythm disturbances are common findings in HIV-positive patients with myocarditis or heart muscle disease, and ectopic beats, ventricular tachycardia, and sudden death have all been reported.

\section{Pericarditis and Pericardial Effusion}

Pericarditis and pericardial effusion were the commonest cardiac abnormalities found in early HIV/AIDS autopsy studies and remain a significant problem in Africa, where the largest number of HIV/AIDS patients are found.2,3 Small effusions are still found frequently in patients with heart failure or malignant infiltration, but cardiac tamponade may occur rarely. The finding of cardiomegaly on chest radiography should prompt early echocardiographic assessment. Clinically significant pericardial effusions are usually caused by viral or bacterial infection or malignant infiltration, particularly with Kaposi sarcoma or non-Hodgkin lymphoma (NHL). Pericarditis caused by M. tuberculosis or Mycobacterium avium-intracellulare infection is a pressing problem in Africa but has also been reported as the first manifestation of AIDS in Europe. Other unusual pathogens, including Nocardia asteroides and herpes simplex virus, should be considered along with $\mathrm{CMV}$, which remains prevalent in the HIV/AIDS population often without a definite 
anatomical site of infection. Appropriate antituberculous and antiviral therapies may therefore be helpful in this situation. Culture of pericardial biopsy or fluid from symptomatic effusions may also be also useful in identifying treatable opportunistic infections or malignancy.

\section{Myocarditis}

The prevalence of myocarditis in HIV/AIDS varies from 53\% in the pre-HAART era to much lower levels today in the developed world. Myocarditis can be precipitated by a variety of viral infections, and the inflammatory reaction can progress even after virus is no longer evident in the heart. An immune reaction, either to viral antigen or to altered myocardial protein, may precipitate myocardial necrosis and inflammatory cell infiltration. However, simple histopathologic methods alone may be insufficient to exclude the diagnosis of myocarditis in AIDS patients. Infiltrating CD8 and CD45 lymphocytes have been found in association with increased MHC class I antigen expression in histologically normal endomyocardial biopsies from HIV-positive patients with cardiac failure. ${ }^{8}$ There are several hypotheses regarding the cause of myocarditis in AIDS, including primary HIV myocarditis, secondary HIV myocarditis, opportunistic infection, and autoimmunity.

\section{Primary HIV Infection of the Myocardium}

The virus HIV per se has neither been universally accepted nor unambiguously proven a causative agent of myocarditis in AIDS. HIV-1 gains entry into cells through binding between its envelope glycoprotein group 120 and CD4 receptors, which are found on T4 (helper) lymphocytes and some other cell types. Although HIV can infect monocytes or macrophages and myocardial interstitial cells, evidence proving that HIV can infect human cardiac myocytes, which do not possess CD4 receptors, is less clear. HIV gene sequences have been detected by PCR in microdissected endomyocardial biopsies from HIV-positive patients, some of whom had cardiac symptoms.28 HIV has also been shown to gain entry into the human fetal cardiac myocyte by ingestion through a specific Fc receptor, and it remains possible that this or other unidentified mechanisms may promote HIV entry into the myocyte and facilitate a primary HIV myocarditis.29

\section{Secondary HIV Myocarditis}

Immune responses are implicated in Chagas' cardiomyopathy in which noninfected myocytes are damaged by the host response to Trypanosoma cruzi. Interstitial lymphocytes and macrophages may form contact with myocytes, causing a focal loss of basement membrane through a local reaction. A similar process may be involved in the pathogenesis of HIV myocarditis. Proteolytic enzymes released through HIV replication in the interstitium could also damage myocytes. Such "innocent bystander destruction" may be particularly relevant to the myocardium because increased numbers of infected interstitial cells have been found in HIV-positive subjects with active myocarditis. The HIV envelope glycoprotein group 120 can induce tumour necrosis factor (TNF) expression from macrophages. Cytokine IL-6, which has some effect on immune response and viral replication in murine myocarditis models, has been found in excess in a small number of HIV-positive patients with biopsy-proven myocarditis. Therefore, just as cytokines may have a role in the development of congestive heart failure in absence of HIV/AIDS, they may also be important in the course of HIV/AIDS myocarditis and cardiomyopathy.

\section{Myocardial Opportunistic Infections in HIV/AIDS}

Autopsy has confirmed a variety of opportunistic infections of the myocardium in patients with AIDS. Infectious agents have included Toxoplasma gondii in the hearts of adults and children, Cryptococcus spp., CMV, Candida spp., Pneumocystis carinii, Microsporidium spp., Histoplasma capsulatum, atypical mycobacteria, and Aspergillus organisms involving the myocardium. Most of these have been part of a disseminated infection and are infrequently associated with localized myocarditis. Acute Chagas' myocarditis in patients with AIDS also has been reported and may be associated with a more frequent rate of myocarditis in up to $30 \%$ of cases in AIDS patients. However, a clear causative link between opportunistic infection and myocarditis in AIDS has yet to be established.

\section{Clinical Features of Myocarditis in HIV/AIDS}

Myocarditis can be diagnosed clinically based on symptoms and physical findings, although this is often difficult in HIV/AIDS patients. The symptoms are protean and include fatigue, dyspnoea, and pleuritic chest pain, which may wrongly be ascribed to other conditions. The finding of an unexplained tachycardia, a third heart sound, or a friction rub should alert the physician to the possibility of the myocarditis and guide investigation. The electrocardiogram (ECG) may be helpful, possibly demonstrating nonspecific conduction defects, repolarization abnormalities, and ST-T-wave changes. The chest radiograph may be normal or suggest cardiac enlargement with pulmonary congestion. Echocardiography is usually nondiagnostic but may show hyperdynamic LV function in HIV-positive children with myocarditis or occasionally LV dyskinesia in adult HIV/AIDS patients. The utility of a myocardial biopsy remains unclear in this setting.

\section{Endocarditis}

Endocarditis is the principal endocardial lesion identified pathologically in HIV/AIDS, and both infectious and noninfectious forms have been described. At present, the prevalence of non-infectious endocarditis is lower than originally reported, and HIV itself has not emerged as a significant risk factor for infectious endocarditis. Nonetheless, these conditions remain a potential cause of significant morbidity.

\section{Nonbacterial Thrombotic Endocarditis}

Marantic or nonbacterial thrombotic endocarditis (NBTE) is a condition in which friable clumps of platelets and red blood cells adhere to the cardiac valves. Unlike bacterial endocarditis, these lesions are not infective and show no evidence of an inflammatory reaction. NBTE was found in a 
homosexual man receiving chemotherapy for Kaposi sarcoma in 1982 and had been reported in approximately 10\% of adult HIV/AIDS patients in autopsy series from that time.10 The pathogenesis of NBTE is not fully understood, but hypercoagulability, immune complex deposition, or specific vitamin deficiency may be important in conjunction with endothelial damage from intracardiac catheters or injected particulate matter. Any heart valve may be affected, and multiple lesions are frequently found on different valves. 10 Systemic thromboembolism is a common sequela and contributes to the morbidity and mortality associated with the condition. However, the incidence of NBTE in HIV/AIDS appears to be decreasing. This may be surprising in face of the growing interest in the cardiac manifestations of HIV/AIDS and continuing echocardiographic surveillance. The reasons for this decline are not clear but may be related to improvements in nutrition and HIV/AIDS therapy, including HAART.

\section{Infective Endocarditis}

The immunologic abnormalities associated with HIV render patients susceptible to bacterial infections. Despite this, there are few reports of infective endocarditis in association with HIV/AIDS, and infective endocarditis rarely complicates HIV infection in absence of the setting of injecting drug use. Bacterial endocarditis has a similar presentation to nonHIV/AIDS patients but runs a more fulminant course in the late stages of HIV/AIDS. However, asymptomatic HIV infection appears to have little effect on the susceptibility to or the mortality from infective endocarditis. As with infective endocarditis in HIV-negative intravenous drug users, the most common valve involved is the tricuspid valve, although left-sided valves may also be affected. The most frequently isolated organisms are S. aureus and Streptococcus viridans, but Aspergillus fumigatus, Pseudallescheria boydii, and other forms of fungal endocarditis may occur in end-stage AIDS. Clearly, there is a need for adequate bacteriologic investigations in cases of suspected infective endocarditis in HIV-positive individuals just as for the non-AIDS population. However, the initial choice of antimicrobial treatment may have to be adjusted, particularly if fungal endocarditis is a suspected complication of end-stage AIDS. Valvular heart surgery has been used in cases of infective endocarditis in HIV-positive intravenous drug users.

\section{Pulmonary Hypertension and Right Ventricular Dysfunction}

RV dysfunction may occur as part of HIV-related heart muscle disease and in these circumstances should be treated as described previously. Isolated RV dysfunction without pulmonary hypertension is of unknown significance. Therefore, bronchopulmonary infections should be treated aggressively, and intravenous drug use, which may result in microvascular pulmonary emboli, should be discouraged. Isolated pulmonary hypertension is a rare and serious complication of HIV infection, although it occurs more frequently than does primary pulmonary hypertension in the non-HIV population. The condition has a grave prognosis with an approximate $50 \%$ survival at 1 year despite having little correlation with CD4 counts or history of pulmonary infections. Experimental data suggest that both protease inhibitors and HIV gene products may be implicated in the development of pulmonary hypertension in HIV/AIDS through downregulation of in vitro endothelial nitric oxide synthase and pulmonary vasomotor dysfunction. ${ }^{11}$ Although pulmonary hypertension in HIV/AIDS may also be related to the action of viral proteins or the action of cytokines on the endothelial cell, characteristic pulmonary arteriopathy is found in HIV-related pulmonary hypertension. The pathological lesions include intimal fibrosis remodelling and plexiform lesions, confirming its similarity to primary pulmonary hypertension and thus guides possible therapies. Right heart catheterization may be worthwhile in HIV/AIDS patients. Continuous or intermittently delivered home oxygen therapy, calcium channel antagonists, and nitric oxide therapy may be as useful for HIV/AIDS patients as for primary pulmonary hypertension patients, although their use is largely unproven. Curiously, despite concerns about possible effects on pulmonary endothelial function, HAART itself may be beneficial in terms of outcome from pulmonary hypertension. Agents such as oral bosentan, intravenous epoprostenol, subcutaneous treprostinil, or sildenafil could improve feelings of well-being without necessarily altering the patient's prognosis. However, sildenafil and bosentan should be used with care in patients on HAART because of potential drug interactions.

\section{Cardiac Tumours}

Primary and metastatic tumours of the heart are relatively uncommon, but the advent of HIV/AIDS has changed both their prevalence and their appreciation in some ways. Kaposi sarcoma is the most common AIDS-related neoplasm, and in contrast to the classical dermatologic form of the disease, there is often widespread and potentially fatal visceral involvement in individuals with HIV/AIDS. Therefore, although Kaposi sarcoma involving the heart is rare outside this setting, it is now well recognized in patients with AIDS. The prevalence of cardiac Kaposi sarcoma in HIV/AIDS appears to have decreased significantly since early reports. Kaposi sarcoma is an endothelial cell neoplasm and in HIV/AIDS shows a predilection for the subpericardial fat around coronary arteries, where it may infiltrate. The parietal pericardium or myocardium may also be involved. Kaposi sarcoma is not usually associated with symptoms of cardiac dysfunction. Nonetheless, fatal tamponade associated with Kaposi sarcoma has been reported, and heart failure without ventricular dilatation may occur in cases with extensive myocardial infiltration. Kaposi sarcoma may be treated with daunorubicin, doxorubicin, or related anthracyclines, although care is required because these drugs may themselves cause a toxic cardiomyopathy that may occur some years after initial treatment.

\section{Cardiac Lymphoma in HIV/AIDS}

Primary cardiac lymphoma is extremely rare in HIV-negative individuals, accounting for fewer than $10 \%$ of all primary malignant cardiac tumours. Disseminated lymphoma may involve the myocardium more frequently but usually only as part of widespread tumour involvement. However, both 
patterns of malignant cardiac involvement occur more commonly in individuals with HIV/AIDS. NHL may involve the pericardium or myocardium in patients with HIV/AIDS, and echocardiography is useful to detect any related intracavitary masses or concomitant pericardial effusions. Radionuclide and magnetic resonance imaging scans may be required to detect more diffuse cardiac involvement. In contrast to Kaposi sarcoma, cardiac lymphoma commonly give rise to clinical symptoms of tamponade, heart failure, and conduction abnormalities and should be considered in AIDS patients whose cardiovascular symptoms progress rapidly. Primary lymphoma involving the heart alone is uncommon in AIDS, but surgical resection of a right atrial lymphoma was reported in an HIV-positive patient with limited short-term success.

\begin{abstract}
Vasculitis
Vascular changes, particularly vasculitis and endothelial cell structural changes, have been documented in individuals with HIV/AIDS. Examination of aortic endothelial cells from HIV-infected patients showed a disturbed intima, increased leukocyte adherence, and upregulation of vascular cell adhesion molecules. This indicates profound and repeated activation of aortic endothelial cells in HIV/AIDS, and it is possible that $\backslash$ some HIV structural proteins may play an integral role in the process. The clinical importance of such findings is not clear, and lesions affecting small- and mediumsized arteries are usually only evident by microscopy. However, fusiform aneurysms of the right coronary artery were found in a child who died suddenly. These lesions were histologically distinct from other arteriopathies, but other vascular abnormalities in children have been reported in the brain, thymus, spleen, and lymph nodes. Kawasaki disease, which may have a similar presentation, should be considered as a differential diagnosis that is excluded pathologically.
\end{abstract}

\section{Autonomic Dysfunction}

Autonomic dysfunction is common in patients with HIV infection, and this may predispose to syncopal events and dysrhythmias because of excessive sympathetic tone. Conduction abnormalities and arrhythmias have been demonstrated in HIV-positive children with ECG abnormalities, possibly related to small vessel vasculitis, neural tissue fibrosis, or myocarditis. At the other extreme, as the age of HIV-infected people treated with HAART increases, coincidental cardiac rhythm disturbances will become more common. Atrial fibrillation and sick sinus syndrome with symptomatic bradycardia requiring permanent pacing have been described in HIV/AIDS patients. Caution is required for the use of amiodarone and digoxin for rhythm or rate control in such circumstances because further potential drug interactions exist.

\section{CONCLUSIONS}

It seems likely that as survival increases with improved therapy, more HIV/AIDS patients will be seen by cardiologists, and indeed the number of reports of cardiovascular problems in HIV/AIDS is increasing.
Nonetheless, cardiovascular disease in individuals with HIV/AIDS is also becoming increasingly recognized in the developing world perhaps as antiretroviral therapy becomes more readily available there. Heart disease may be overlooked in HIV-positive patients because symptoms of breathlessness, fatigue, and poor exercise tolerance are frequently ascribed to other conditions associated with HIV infection. Echocardiographic assessment of HIV/AIDS patients is extremely useful and can be used easily to identify those cardiac conditions common in HIV-positive patients that may be associated with a poor outcome, including pericardial effusion, left ventricular (LV) systolic dysfunction or heart muscle disease, and intracardiac masses. Echocardiography may also provide useful information on the appearance of the right ventricle (RV), provide an indirect assessment of pulmonary pressures, and reveal regional wall motion abnormalities suggestive of CAD. It has been suggested that any HIV-positive patient who is at high risk of developing or who demonstrates any potential clinical manifestation of cardiovascular disease should have a baseline echocardiogram performed. Thereafter, serial echocardiography should be performed every 1 to 2 years. ${ }^{12}$ Clearly, more aggressive monitoring may be guided by the cardiologist on discovery of abnormalities or in those patients with significant, potentially cardiotropic viral infections or unexplained pulmonary symptoms.

\section{REFERENCES}

[1] Vitoria M, Granich R, Gilks CF, et al. The global fight against HIV/AIDS, tuberculosis, and malaria: current status and future perspectives. Am J Clin Pathol 2009;131(6):844-8.

[2] Ntsekhe M, Mayosi BM. Cardiac manifestations of HIV infection: an African perspective. Nat Clin Pract Cardiovasc Med 2009;6(2):120-7.

[3] Reuter H, Burgess LJ, Schneider J, et al. The role of histopathology in establishing the diagnosis of tuberculous pericardial effusions in the presence of HIV. Histopathology 2006;48(3):295-302.

[4] Vittecoq D, Escaut L, Chironi G, et al. Coronary heart disease in HIV-infected patients in the highly active antiretroviral treatment era. AIDS 2003;17(Suppl 1):S70-6.

[5] Nieto FJ. Infective agents and cardiovascular disease. Semin Vasc Med 2002;2(4):401-15.

[6] Currie PF, Jacob AJ, Foreman AR, et al. Heart muscle disease related to HIV infection: prognostic implications. Br Med J 1994;309(6969):1605-7.

[7] de Larranaga GF, Forastiero RR, Carreras LO, et al. Different types of antiphospholipid antibodies in AIDS: a comparison with syphilis and the antiphospholipid syndrome. Thromb Res 1999;96(1):19-25.

[8] Herskowitz A, Wu TC, Willoughby SB, et al. Myocarditis and cardiotropic viral infection associated with severe left ventricular dysfunction in late-stage infection with human immunodeficiency virus. J Am Coll Cardiol 1994;24(4):1025-32.

[9] Moorthy LN, Lipshultz SE. Cardiovascular monitoring of HIV-infected patients. In: Lipshultz S, ed. Cardiology in AIDS. New York NY: Chapman \& Hall 1998:345-84. 
[10] Cammarosano C, Lewis W. Cardiac lesions in acquired immune deficiency syndrome (AIDS). J Am Coll Cardiol 1985;5(3):703-6.

[11] Wang X, Chai H, Lin PH, et al. Roles and mechanisms of human immunodeficiency virus protease inhibitor ritonavir and other anti-human immunodeficiency virus drugs in endothelial dysfunction of porcine pulmonary arteries and human pulmonary artery endothelial cells. Am J Pathol 2009;174(3):771-81.

[12] Volberding PA, Murphy RL, Barbaro G, et al. The Pavia consensus statement. AIDS 2003;17 Suppl 1:S170-9. 